\title{
Annual Scientific Report for DE-FG03-02NA00063 \\ Coherent imaging of laser-plasma interactions using XUV high harmonic radiation
}

\author{
Prof. Henry C. Kapteyn \\ JILA, University of Colorado at Boulder, Boulder, CO 80309-0440 \\ Phone: (303) 492-8198; FAX: (303) 492-5235; E-mail: kapteyn@jila.colorado.edu
}

\author{
1. Personnel supported by and collaborating on grant to date (Years 1 and 2) \\ Professor Henry Kapteyn (no salary support) \\ Professor Margaret Murnane (no salary support) \\ Ariel Paul, graduate student (full salary support) \\ Ryan Anderson, graduate student (partial salary support) \\ Dr. Sterling Backus, Research Scientist (partial salary support) \\ Daisy Raymondson, graduate student (full salary support) \\ Dr. Oren Cohen (partial salary support since has partial fellowship for Yr 1 of postdoc) \\ James Holtsnider (partial salary support)
}

\section{Financial status of grant}

We are currently in Year 3 of this grant. We expected to have the remaining grant funds expended by the end of 2005. We have several pieces of equipment associated with the project, including an x-ray CCD camera and vacuum pumps, that need to be repaired (or replaced if repair is not possible) due to the difficult environment associated with plasma imaging.

3. Highlights of Progress to Date: We report four main highlights to date -

1. Our group published 12 papers, received 3 major national awards, and obtained one patent in work partially or primarily supported by the prior grant.

2. Two people (one M.S. student and one postdoc) from our group took positions at DP Laboratories - Dr. Erez Gershgoren at LANL and Jason Schmidt at LLNL.

3. The high-power femtosecond laser system designs developed in our group during the past two grant periods under partial DOE/NNSA support are now in use in 3 DP Research Labs.

4. We recruited two new students, supervised by a new a postdoctoral fellow for this work.

\section{Demonstration of fully spatially coherent EUV beams at $13 \mathrm{~nm}$ in plasmas}

In recent work, we demonstrated full spatial coherence of extreme ultraviolet (EUV) beams generated by the process of high harmonic upconversion of a femtosecond laser, at wavelengths around $11 \mathrm{~nm}$ and $13 \mathrm{~nm}$. It is important to note that this work to demonstrate full coherence around $13 \mathrm{~nm}$ was not merely an extension of our previous work where we demonstrated full spatial coherence at wavelengths around $30 \mathrm{~nm}$. The new work required a much more intensive experimental and theoretical study of the interaction of intense ultrashort laser beams with rapidly ionizing plasmas, and several unexpected results were obtained.

To obtain such a high-flux, fully coherent beam at $13 \mathrm{~nm}$, we first needed to improve phase matching techniques in ionized plasmas so that we could generate this wavelength more efficiently - even just in order to characterize the radiation. Experimentally, in work published in Nature and Science, we demonstrated quasi phase matching of high harmonic radiation using 
modulated waveguides to contain the gas medium, to extend the observable, bright, harmonic orders to shorter wavelengths.

The next step was to measure the spatial coherence of the EUV beams from these modulated waveguides, particularly at very short wavelengths around $11-13 \mathrm{~nm}$ that would enable higher spatial resolution using more efficient EUV optics. At high laser intensities and ionization levels, the laser beam is refracted by a plasma and continually re-guided by the modulations on the hollow modulated waveguide. Therefore, it was not at all certain that the output EUV beams would exhibit full spatial coherence compared with that obtained at wavelengths around 30nm where the gas is not highly ionized. The degree of spatial coherence can be determined by measuring the fringe visibility in a Young's double pinhole experiment as a function of pinhole separation across the EUV beam. Using this approach, in work published in Optics Letters and Applied Physics Letters, we demonstrated that phase matched and quasi-phase matched highharmonic generation in modulated waveguides does indeed produce EUV beams with full spatial coherence at wavelengths from $30 \mathrm{~nm}-13 \mathrm{~nm}$, provided that relatively long waveguides with lengths $>6 \mathrm{~cm}$ are used.

Figure 1 shows sample interferograms and their lineouts for $1 \mathrm{~mm}$ EUV beams centered at 30nm and $13 \mathrm{~nm}$, diffracted by pinhole pairs at separations of $150 \mu \mathrm{m}$ and $250 \mu \mathrm{m}$. Because broad bandwidth combs of 5-7 harmonics were used for this measurement, the fringe contrast is $100 \%$ only at the center, since each harmonic gives rise to a slightly different interference pattern on the CCD camera. This series of experiments demonstrated the highest spatial coherence values ever measured at such short wavelengths from any source without spatial filtering. Therefore, HHG-based EUV sources will be useful for applications in metrology, coherent imaging, nonlinear spectroscopies, and microscopy.

Figure 1 (right) also shows the measured fringe visibility (spatial coherence) as a function of waveguide length for EUV beams generated in 40 torr of Argon. It is clear that in order to generate fully spatially coherent extreme-ultraviolet (EUV) beams using HHG, the driving laser beam must be guided over relatively long interaction lengths $(\sim 10 \mathrm{~cm})$. To understand the evolution of the laser pulse and the EUV beam in the plasma contained within a modulated waveguide, our theory collaborator, Dr. Ivan Christov, used a fully space-time model to predict the propagation of an intense laser pulse through a multiply-ionizing plasma in a guided geometry. This model takes into account contributions to the laser and EUV pulse propagation due to diffraction, dispersion of the neutral atoms, dispersion due to the time-dependent plasma density, and loss due to ionization. Also, by applying appropriate boundary conditions for the hollow waveguide, the model can include the additional negative dispersion that is present in the guided geometry. When comparing the waveguide with a free-space geometry (i.e. HHG in a jet), the Guoy phase shift is automatically included in the calculations. Using this model we were thus able to follow the processes of phase matching in the hollow waveguide, and its dependence on the key parameters of the experiment. In particular, we can compare the spatial properties of the laser and EUV beams both in the free-focused and guided-wave geometries, shown in Fig. 2. Our radiation and plasma models were able to explain why full spatial coherence was observed only for long interaction lengths in a guided geometry.

This work has had four important outcomes to date: 1) It uncovered a new regime of guiding a laser beam in a plasma-filled waveguide, where a stable eigenmode can be formed even in a plasma-filled waveguide. 2) As a result of being able to support a stable laser eignenmode in the plasma-filled waveguide, the generated EUV beams were perfectly spatially coherent. 3) In related work, also because of the support of a stable laser eigenmode in a plasma-filled waveguide, very high-order harmonic generation from ions was observed. 4) Finally, in other related work, a new pulse compression mechanism was identified, where the laser pulse emerges from the waveguide with a shorter pulse duration than when it entered without any need for dispersion compensation. The shorter pulse duration was due to rapid phase modulation in a 
rapidly ionizing plasma, which rapidly changes the index of refraction. When this effect is combined with plasma refraction and guiding in a waveguide, the leading edge of the laser pulse steepens, and the pulse duration is reduced by a factor of $2-3$, from $30 \mathrm{fs}$ to $13 \mathrm{fs}$. This combined work also has exciting new possibilities for uncovering new ultrafast plasma physics, as well as having consequences for coherent EUV source technologies.

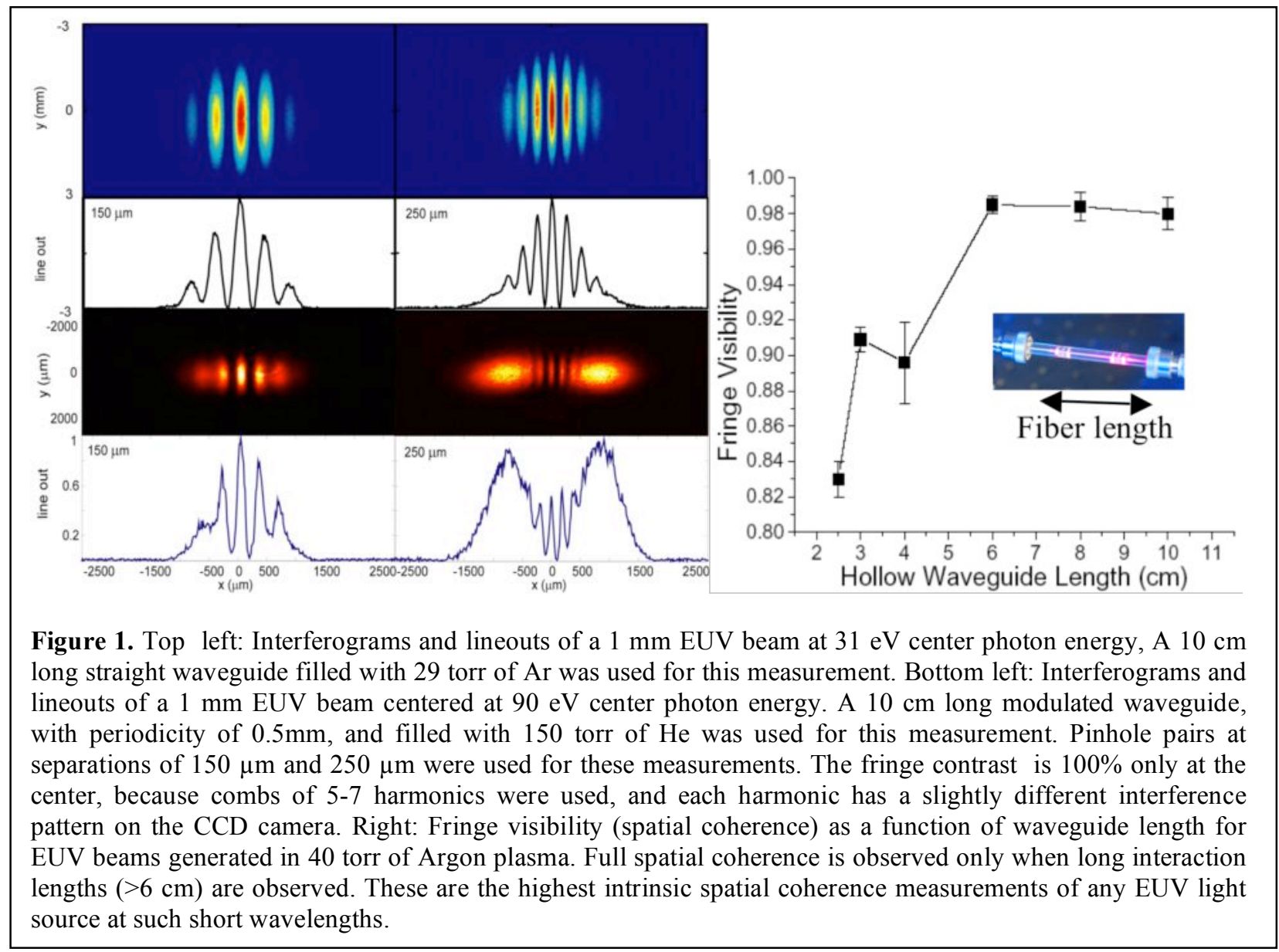

\section{High-resolution static and dynamic imaging using an EUV microscope}

The ultimate goal of the current project was to apply EUV sources to high-resolution imaging of high-density, warm or turbulent plasmas. This required development of a long-working-distance EUV microscope based on multilayer optics. We first implemented high-resolution static imaging of small objects, using the set-up shown in Figure 3. A static grid with $10 \mu \mathrm{m}$ thick wires was inserted instead of the droplet source, and positioned about $50 \mathrm{~cm}$ from the EUV source. Two multilayer mirrors were used to produce a x50-magnified image on an x-ray CCD camera. The first mirror had a focal length of $50 \mathrm{~cm}$, and was positioned about $58 \mathrm{~cm}$ from the object, at a magnification of $x 7$. Figure 4 shows the magnified image of a 10-micron grid taken at a magnification of x50. Changing the distance between the EUV mirrors and between the mirrors and the CCD camera varies the magnification. The smallest resolvable feature size in theory is $13.5 \mu \mathrm{m} / 50$ or $270 \mathrm{~nm}$. However, in these images, we were limited by the numerical aperture of the system, and therefore the actual resolution was $\sim 1$ micron. Further optimization of the setup should yield even higher resolution in the near future. 


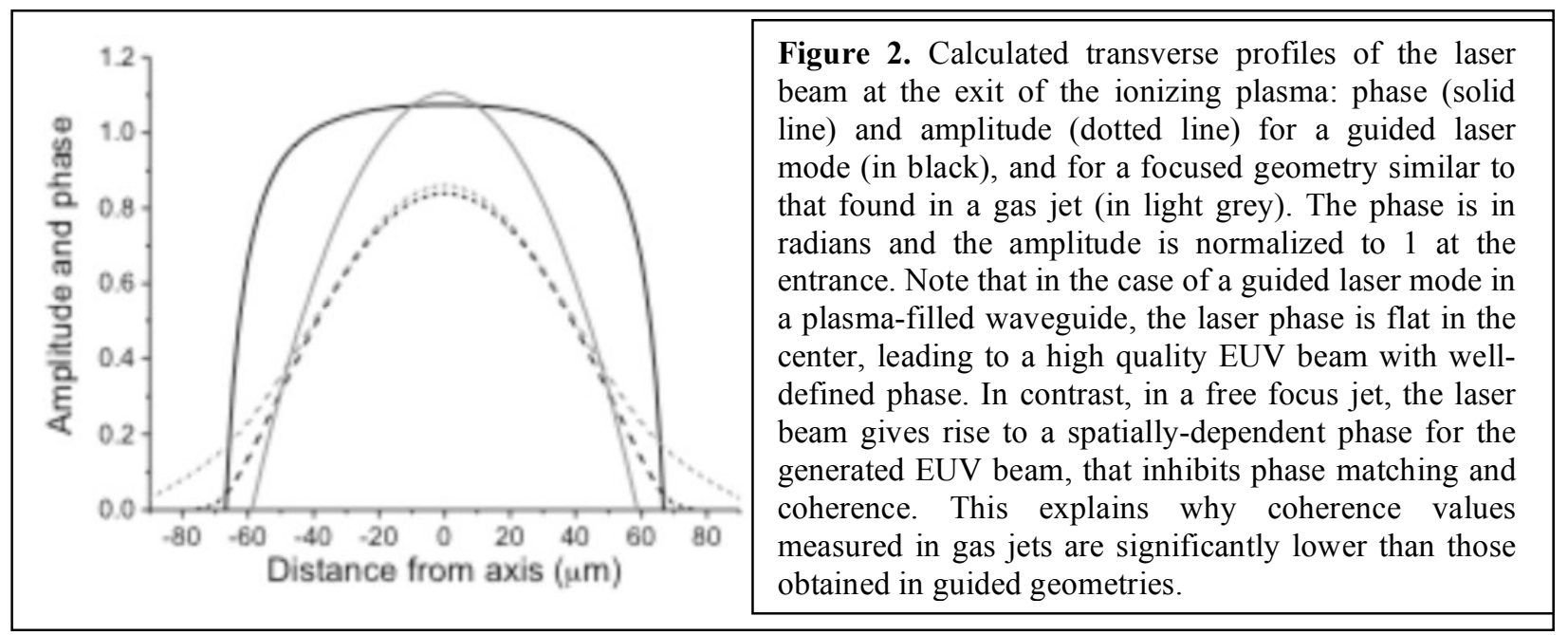

For dynamic imaging of plasmas, we developed a new synchronized droplet source that is now in operation. Droplet plasmas provide us with more opportunities for imaging turbulent and colliding plasmas than simply imaging a gas or liquid jet. We obtained the first sequence of images of ultrafast plasma dynamics taken using coherent EUV light at a wavelength of 30nm. Laser pulses at a repetition rate of $1-2 \mathrm{kHz}$ generated the EUV beams used for illumination, as well as for perturbing the water droplet to observe dynamics. In a series of images shown in the lower image of Fig. 4, three droplets can be seen (top to bottom) in each frame, with the center drop illuminated by $\sim 100$ microjoules of energy from the ultrafast laser. With varying pumpprobe delay, the expansion of the plasma can be observed. These are stroboscopic images, with each pump-probe delayed image taken over many thousands of laser shots. The jitter in droplet position is estimated from coherent artifacts in the data to be on order of 100nn.

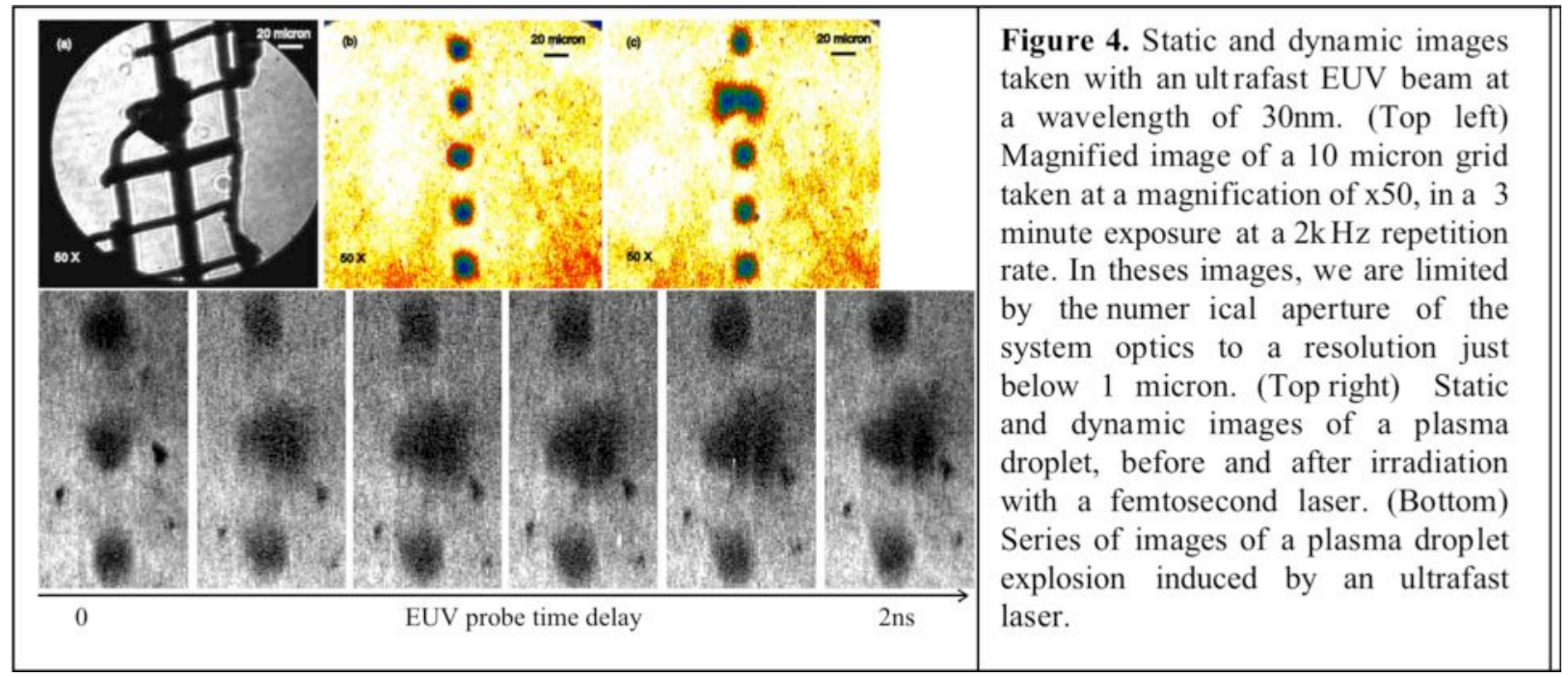

This series of experiments has proven the basic feasibility of the proposed experiments and shown that we should be able to observe turbulent and colliding plasmas by illuminating two droplets at once. However, to image the critical density fronts and to gain more detailed information, we need to increase the spatial resolution of these images. The current series of experiments ran into a number of challenges, including understanding timing-stabilization of the droplet source, and (most significantly) developing alignment and focusing methodologies for a totally vacuum-enclosed imaging system spanning rather large ( $\sim$ meters end-end) distances 
between the mirrors (necessitated by the large working distance requirement in the case of plasma imaging). We will continue with these experiments during the current grant period, and then expand on this work in new directions, if this grant is renewed.

\section{Future Work}

Our experiments to date have uncovered new physics and applications involving the interaction of intense laser pulses with low-density plasmas in guided geometries. We also demonstrated in initial experiments that we can make detailed studies of plasma dynamics in a cumulative, "stroboscopic" mode. In the long term, if this grant is renewed, we plan to expand upon our studies of the interaction of intense, ultrashort, laser pulses in under-dense plasmas, to understand how to control this interaction, for example to create periodically modulated plasma and ionization profiles. We also plan to explore new experimental techniques for the study of high-density plasmas. Specifically, the use of transient grating techniques to study the dynamics of warm-dense plasmas promises to give data with unprecedented detail in expansion dynamics. Finally, we plan to expand our work in a new direction, in collaboration with Dr. Jim Glownia at Los Alamos National Laboratory, to study phase transitions in materials such as $\mathrm{VO}_{2}$ and cerium, using linear and nonlinear EUV spectroscopies as a probe.

\section{Publications as a Result of Prior Award}

1. Henry C. Kapteyn, Margaret M. Murnane and Ivan P. Christov, "Coherent X-Rays from Lasers: Applied Attosecond Science", invited article, Physics Today, page 39 (March 2005).

2. E. A. Gibson, X. Zhang, T. Popmintchev, A. Paul, N. Wagner, A. Lytle, I.P. Christov, M.M. Murnane and H.C. Kapteyn, "Extreme Nonlinear Optics: Attosecond Photonics at Short Wavelengths", invited paper, to be published in IEEE JSTQE (2005).

3. X. Zhang, D. Raymondson, A. S. Sandhu, S. Backus, M. M. Murnane, H. C. Kapteyn, and A. R. Libertun, "Coherent Imaging of Laser Plasma Interactions using High Harmonic EUV Light," in Proceedings of the International Conference on Ultrafast Phenomena, Springer Chemical Physics series, to be published (2005).

4. X. Zhang, A. R. Libertun, A. Paul, E. Gagnon, S. Backus, I. P. Christov, M. M. Murnane, H. C. Kapteyn, R. A. Bartels, Y. Liu, and D. T. Attwood, "Highly coherent light at $13 \mathrm{~nm}$ generated by use of quasi-phase-matched high-harmonic generation," Optics Letters 29, 1357 (2004).

5. A. Libertun, X. Zhang, A. Paul, E. Gagnon, T. Popmintchev, S. Backus, M. Murnane, H. Kapteyn, I. Christov, "Design of fully spatially coherent extreme-ultraviolet light sources," Appl. Phys. Lett. 84, 3903 (2004.)

6. X. Zhang, D. Raymondson, A. S. Sandhu, S. Backus, M. M. Murnane, H. C. Kapteyn, and A. R. Libertun, "High-resolution imaging system using a tabletop extreme ultraviolet source," in Proceedings of the SPIE, vol. 5534. Bellingham, WA: SPIE, 2004, pp. 47-52.

7. Ariel Paul, Randy Bartels, Ivan Christov, Henry Kapteyn, Margaret Murnane, Sterling Backus, "Multiphoton photonics: quasi phase matching in the EUV", Nature 421, 51 (2003).

8. R. Bartels, A. Paul, S. Backus, H Kapteyn, M. Murnane, "EUV Photonics: Quasi Phase matching at Short Wavelengths", OSA Proc. Ultrafast Phenomena XIII (Springer Series in Chemical Physics), page 51 (2003).

9. R. Bartels, S, Backus, A. Paul, H Kapteyn, M. Murnane, "Determination of the HHG spectrum by measuring the mutual coherence", OSA Proc. Ultrafast Phenomena XIII (Springer Series), page 55 (2003).

10. R. Bartels, S. Backus, A. Paul, H Kapteyn, M. Murnane, Y. Liu, D. Attwood, C. Jacobsen "Fully spatially coherent EUV source", OSA Proc. Ultrafast Phenomena XIII (Springer Series), page 66 (2003).

11. R. Bartels, A. Paul, M. M. Murnane, H. C. Kapteyn, and S. Backus, "Absolute determination of the wavelength and spectrum of an EUV beam using a Young's double slit measurement”, Optics Letters 27, 707 (2002).

12. R. Bartels, A. Paul, H. Green, H. Kapteyn, M. Murnane, S. Backus, I. Christov, Y. Liu, D. Attwood, C. Jacobsen, "Generation of spatially coherent light at extreme ultraviolet wavelengths," Science 297, 376 (2002). 


\section{Honors during Prior Award}

1. Margaret Murnane was elected to the National Academy of Sciences (2004)

2. Randy Bartels (graduate student and postdoc partly supported by grant) received the Lomb Medal of the Optical Society of America (2004)

3. Margaret Murnane was elected Fellow of the AAAS (2003)

\section{Interactions with National Laboratories and Government Agencies}

We have been and will continue to interact with DP personnel as part of this project. In particular, we summarize the following interactions and plans -

1. We have coauthored a number of papers with DOE personnel. (D. Attwood, E. Gullikson from LBL)

2. We attended three DOE and DOD workshops on homeland security, directed energy, and EUV light sources.

3. Our group presented the Physics Colloquium at Los Alamos National Laboratories. We made contact with Dr. Jim Glownia during that visit, and have planned a series of complementary experiments on the same materials using different $\mathrm{x}$-ray and EUV probes. These experiments are discussed in this proposal. Because of the proximity of LANL to Boulder, we planned a series of visits to facilitate interactions.

4. We invited Dr. Antoinette Taylor from Los Alamos National Laboratories to present the Physics Colloquium at Boulder, and to expose our large graduate program $(<214$ students) to work being done at DP Labs. In particular, we have an Optical Science and Engineering Ph.D. program already in place at $\mathrm{CU}$ that requires students to do part of their Ph.D. work outside of CU to broaden their educational experience. This program provides one very convenient mechanism for student interaction with the DP labs. A group of students from physics/chemistry and engineering at CU toured labs at LANL in fall 2004.

5. Our student from our group who is working on this project, Daisy Raymondson, will attend the HEDS (High Energy Density Science) Summer School at Berkeley in August. This workshop will take place in August 2005, and is sponsored by LLNL and Rochester. This student has already worked for one year at LLNL.

6. One student from our group, Jason Schmidt, is now working at LLNL. A postdoc from our group, Dr. Erez Gershgoren, is now working at LANL.

7. We had extensive interactions with scientists from DP Labs who were interested in using the high average power laser technology developed in our labs. Three laser systems will be in use at DP Labs in the near future. 